\title{
Novel Calculation for Cross-Relaxation Energy Transfer Parameter Applied on Thulium Highly-Doped Tellurite Glasses
}

\author{
Masaud Taher ${ }^{\mathrm{a}}$, Hrvoje Gebavi ${ }^{\mathrm{a}}$, Stefano Taccheo ${ }^{\mathrm{a}}$, \\ Daniel Milanese ${ }^{\mathrm{b}}$, and Rolindes Balda ${ }^{\mathrm{c}, \mathrm{d}}$ \\ ${ }^{a}$ College of Engineering - Swansea University, Singleton Park, SA2 8PP, Swansea, UK \\ ${ }^{b}$ Politecnico di Torino, Corso Duca degli Abruzzi10, 10100, Torino Italy \\ ${ }^{c}$ Departamento de Fisica Aplicada I, Escuela Superior de Ingenieria, Universidad del Pais Vasco \\ UPV/EHU, Alda. Urquijo s/n 48013 Bilbao, Spain \\ ${ }^{\mathrm{d}}$ Materials Physics Center CSIC-UPV/EHU and Donostia International Physics Center, 20018 \\ San Sebastian, Spain
}

\begin{abstract}
We investigate the cross relaxation parameter of Tm3+ ions in tellurite glasses over a wide range of concentrations: from $0.36 \mathrm{~mol} \%$ up to $10 \mathrm{~mol} \%$. We propose a new measurement approach based on monitoring the steady-state emission spectra. The proposed method is very simple and allows to measure even very highly doped samples. The crossrelaxation parameter shows a linear dependence with respect to dopant concentration over the full investigated interval and the measured slope is $1.81 \times 10-17 \mathrm{~cm} 3 \mathrm{~s}-1 \mathrm{~mol} \%-1$. The linear dependence suggests a dipole-dipole interaction. Keywords: Rare-earth, Cross-relaxation, Thulium
\end{abstract}

\section{INTRODUCTION}

Among rare-earth doped lasers Thulium laser is an excellent candidate for infrared domain applications thanks to its peculiar and broad emission spectrum at around 1.8 micron [1]. This makes this kind of laser very appealing for several application ranging from precise cut and ablation of biological tissues to LIDAR and sensing applications [2-5]. A further advantage relies on pumping process where cross-relaxation mechanism produce two exited Tm ions out of one pump photon absorbed [6]. For this reason the knowledge of cross-relaxation mechanism is of the outmost importance to properly model and design the laser. So far several methods have been proposed to measure the cross-relaxation parameter, usually based on lifetime measurements [7,8] but also on numerical fitting of fluorescence dynamic versus pump power [9]. In all cases was not possible to define the value of cross-relaxation parameter over a wide range of Tm concentration due to the fact that for very high doping level the pump level is strongly quenched by cross-relaxation and fast detectors are needed. In this paper we investigate the cross-relaxation parameter over a wide range of the doping level up to $10 \mathrm{~mol} \%$. To overcome limitation of previous measurement methods we suggests a new method to calculate the cross-relaxation parameters based on steady-state fluorescence measurement. The investigation was done using a set of sample of Tm-doped tellurite glasses. The choice of this glass host was based on the facts they have the lowest phonon energies $(\sim 750 \mathrm{~cm}-1)$ of all oxides glasses, which lead to increase in optical efficiency and decrease in probability of non-radiative multiphonon decay as well as an excellent rare earth ions solubility comparing with silicate and germinate glasses. The above advantages explains why Tm-doped tellurite glass fibre lasers are of a great interest [10-12].

*m.taher@swansea.ac.uk; phone +44 (0)1792 602465

Optical Components and Materials IX, edited by Shibin Jiang, Michel J. F. Digonnet, J. Christopher Dries, Proc. of SPIE Vol. 8257, 825707 · @ 2012 SPIE · CCC code: 0277-786X/12/\$18 · doi: 10.1117/12.910380 


\section{MODELLING}

Figure 1 energy-level diagram of the $\mathrm{Tm}^{3+}$ ion [9] pumped at $790 \mathrm{~nm}$ to the ${ }^{3} \mathrm{H}_{4}$ level. The ${ }^{3} \mathrm{~F}_{4} \rightarrow{ }^{3} \mathrm{H}_{6}$ emission of the neighboring ion is due to the cross-relaxation process: ${ }^{3} \mathrm{H}_{4},{ }^{3} \mathrm{H}_{6} \rightarrow{ }^{3} \mathrm{~F}_{4},{ }^{3} \mathrm{~F}_{4}$ as indicated in Fig. 1
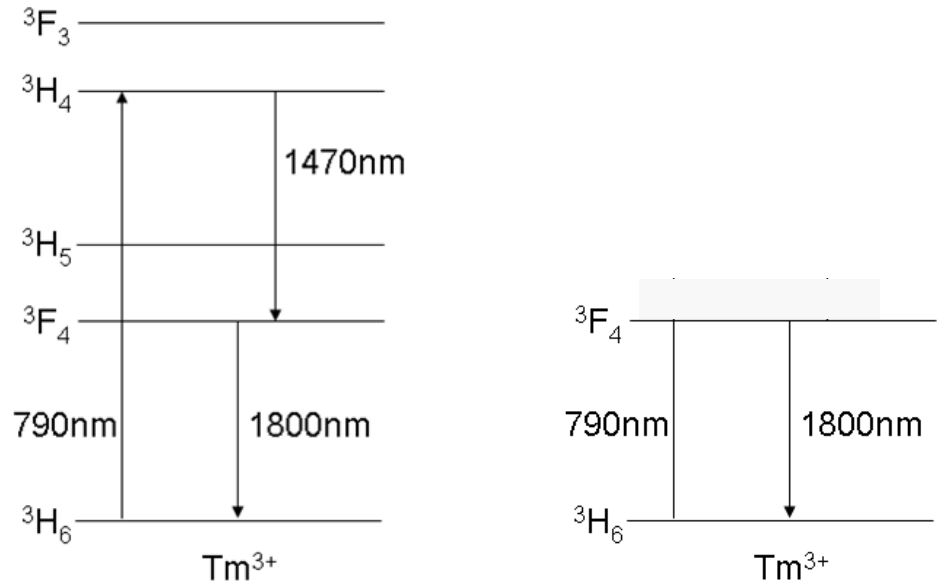

Figure. 1. Simplified energy level diagram of $\mathrm{Tm} 3+$ ion and scheme of the cross-relaxation

The corresponding rate-equation system, considering empty ${ }^{3} \mathrm{H}_{5}$ level, used to analyze our experimental data is:

$$
\begin{aligned}
& \frac{d N_{0}}{d t}=-W_{03} N_{0}+\frac{N_{3}}{\tau_{30}}+\frac{N_{1}}{\tau_{1}}-C_{R} N_{3} N_{0} \\
& \frac{d N_{1}}{d t}=\frac{N_{3}}{\tau_{31}}-\frac{N_{1}}{\tau_{1}}+2 C_{R} N_{3} N_{0} \\
& \frac{d N_{3}}{d t}=W_{03} N_{0}-\frac{N_{3}}{\tau_{30}}-\frac{N_{3}}{\tau_{31}}-C_{R} N_{3} N_{0}
\end{aligned}
$$

where $\mathrm{N}_{0}, \mathrm{~N}_{1}$, and $\mathrm{N}_{3}$ are the populations of $\mathrm{Tm}^{3+}$ ions in the ${ }^{3} \mathrm{H}_{6},{ }^{3} \mathrm{~F}_{4}$, and ${ }^{3} \mathrm{H}_{4}$ levels respectively, $\mathrm{W}_{03}$ is the pump rate $\left(\mathrm{s}^{-1}\right)$ and $\mathrm{C}_{\mathrm{R}}$ the cross relaxation parameter, $\tau_{\mathrm{xy}}$ is the lifetime of the $\mathrm{x}$ to $\mathrm{y}$ transition and $\tau_{\mathrm{x}}$ is the lifetime of level $\mathrm{x}$. Note that $\tau_{3}=\left(\tau_{31}{ }^{-1}+\tau_{30}{ }^{-1}\right)^{-1}$. The $C_{R}$ parameter is a function of the Tm doping level. At steady state the time derivatives in the rate equations are equal to zero. Considering the conservation law of $\mathrm{Tm}^{3+}$ ions populations, we can also write $\mathrm{N}_{\mathrm{t}}=\mathrm{N}_{0}+$ $\mathrm{N}_{1}+\mathrm{N}_{3}$. The methods based on fluorescence lifetime measurements [7,8] uses equation 3 and calculate the crossrelaxation parameter from level 3 lifetime quenching. However lifetime values falls below $1 \mu \mathrm{m}$ for highly-doped samples and measurements of low level signals is difficult. To overcome the limitation of previous methods we propose to investigate the steady-state emission from ${ }^{3} \mathrm{H}_{4}$ and the ${ }^{3} \mathrm{~F}_{4}$ levels. In steady-state condition from Eq. 2 follows:

$$
C_{R}=\frac{1}{2 N_{0}}\left[-\frac{1}{\tau_{31}}+\frac{N_{1}}{N_{3} \tau_{1}}\right]
$$

The ratio between the population of level 1 and 3 can be rearranged by using the Fuchtbauer-Landenburg rule [13]

$$
\sigma_{e, i j}\left(\lambda_{p, i j}\right)=H \frac{\Phi_{i j}\left(\lambda_{p, i j}\right)}{N_{i} \Delta v}
$$


and the relationship that provides the amount of the spontaneous emission [14].

$$
\sigma_{e, i j}\left(\lambda_{p, i j}\right)=K \frac{\lambda_{p, i j}^{4}}{A_{i, j, n o r m} n^{2}\left(\lambda_{p, i j}\right) \tau_{i j}}
$$

where $\sigma_{i j}, A_{i j, n o r m}, \tau_{i j}$ are the emission cross section, the area of emission spectrum normalized to the maximum and the transition radiative lifetime of the transition from level $i$ to level $j$; respectively. The parameters $\mathrm{H}$ and $\mathrm{K}$ are constants independent from the transition, $n$ is the glass refractive index at the transition wavelength; $\Phi_{i j}$ is the number of photons emitted at peak wavelength in the frequency interval $\Delta v, N_{i}$ is the excited ion population, and $\lambda_{p, i j}$ is the emission peak wavelength of the transition labeled as 'ij'. By combining the two above written equations we have:

$$
\frac{N_{i}}{\tau_{i j}} \propto \frac{\Phi_{i j}\left(\lambda_{p, i j}\right)}{\Delta v_{p} \lambda_{p, i j}^{4}} A_{i j, n o r m} n^{2}\left(\lambda_{p, i j}\right)
$$

After substituting into Eq.4 a final form was found:

$$
C_{R}=\frac{1}{2 N_{0} \tau_{31}}[k R-1]
$$

Where $\mathrm{R}$ is the ratio of the emission spectra from and ' $\mathrm{k}$ ' contains all other experimental constant. In our case $\mathrm{k}$ value was 1.33 .

\section{EXPERIMENTS}

All samples had the same host composition $75 \mathrm{TeO}_{2}-20 \mathrm{ZnO}-5 \mathrm{Na}_{2} \mathrm{O}(\mathrm{mol} \%)$, labeled as $\mathrm{TZN}$, and were doped with $\mathrm{Tm}^{3+}$ concentrations ranging from $0.36 \mathrm{~mol} \%$ to $10 \mathrm{~mol} \%[15,16]$. We measured all relevant lifetime parameters by investigation the lowest doping level samples. To calculate the ratio $R$ we excited Tm samples at the wavelength of 785 $\mathrm{nm}$ and we observed the emission from the two transition bands centered at 1.47 and $1.8 \mu \mathrm{m}$, which corresponding to ${ }^{3} \mathrm{H}_{4}$ $\rightarrow{ }^{3} \mathrm{~F}_{4}$ and ${ }^{3} \mathrm{~F}_{4} \rightarrow{ }^{3} \mathrm{H}_{6}$ respectively. Figure 2 shows the experimental set-up. The collecting lens had a relatively small numerical aperture in order to decrease the effect of self-trapping. Focusing was made on glass edge but we tested that focusing up to $0.6 \mathrm{~mm}$ inside the bulk sample did not affect the measured lifetimes.

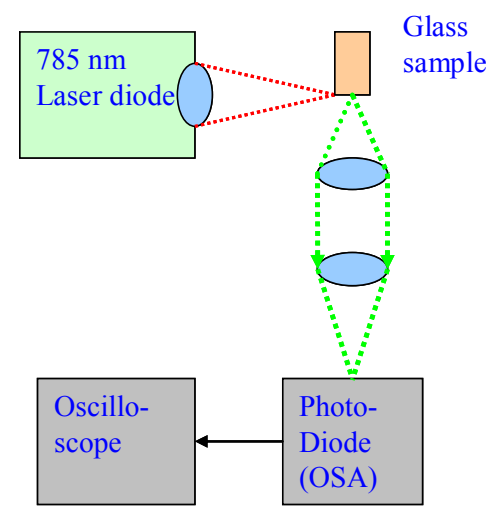

Figure. 2. Schematic of the set-up layout 
The lifetime $\tau_{0}$ for ${ }^{3} \mathrm{H}_{4}$ was $0.37 \mathrm{~ms}$ whilst for ${ }^{3} \mathrm{~F}_{4}$ equal to $2.7 \mathrm{~ms}$. For the samples doped with concentrations higher than $4 \mathrm{~mol} \%$ the lifetime of ${ }^{3} \mathrm{H}_{4}$ level was affected by the response time of our photodiode that is of about few microsecond.

Emission spectra results are shown in Fig. 3 where we normalized all curves to the peak of ${ }^{3} \mathrm{H}_{4} \rightarrow{ }^{3} \mathrm{~F}_{4}$ transition at 1.47 $\mu \mathrm{m}$.

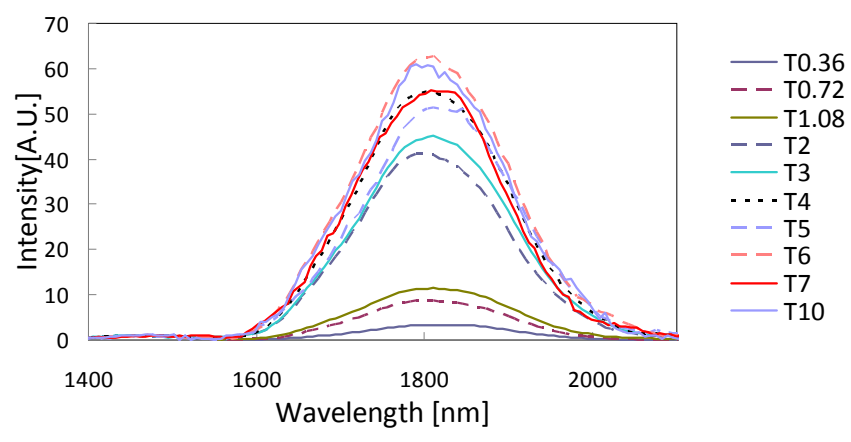

Figure. 3 Emission spectra, inset magnifies the of ${ }^{3} \mathrm{H}_{4} \rightarrow{ }^{3} \mathrm{~F}_{4}$ transition at $1.47 \mu \mathrm{m}$.

The corresponding ratio to be used in Eq.8 is

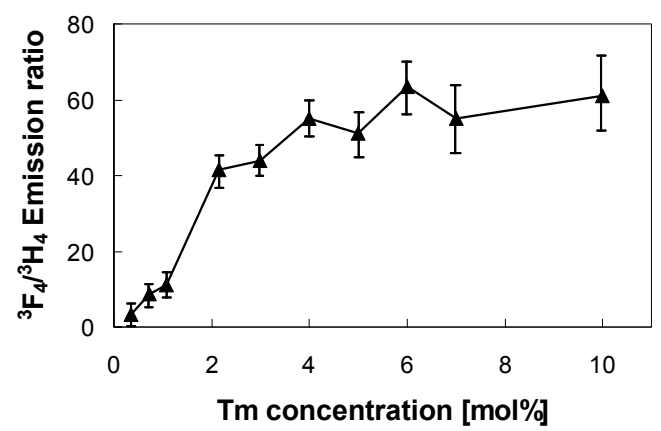

Figure. 4 Ratio between the emission from ${ }^{3} \mathrm{~F}_{4}$ and ${ }^{3} \mathrm{H}_{4}$ levels.

Figure 5 shows the cross-relaxation parameter values calculated by using Eq. 8. We can note a quite clear linear increase even for highest doped samples where, however the slope is slightly reduced.

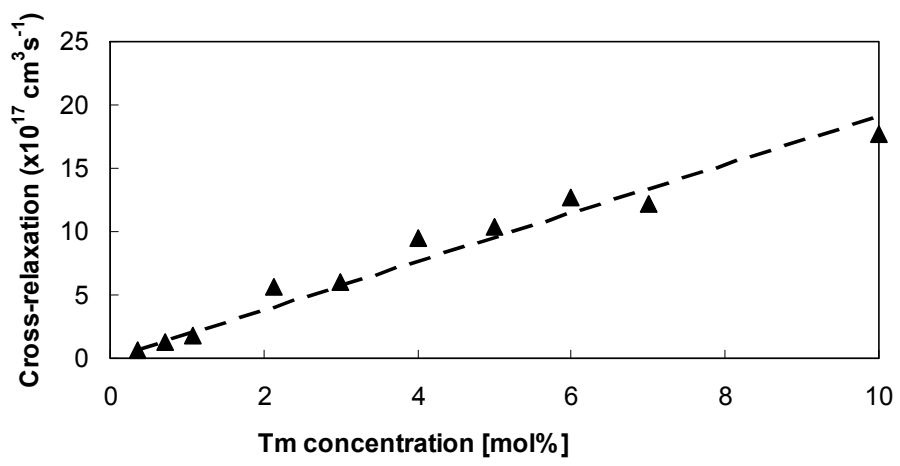

Figure. 5. Cross-relaxation parameter versus Tm concentration. 
From the experimental data the fitted CR is $1.81 \times 10-17 \mathrm{~cm} 3 \mathrm{~s}-1 \%$ mol-1 is obtained. While this value is below the one previously reported in TZN glass [9] it is consisted with the one used for silica fiber modeling [17]. The observed linear dependence on concentration of the cross-relaxation parameter can be explained with the behavior of the concentration quenching of the lifetimes, as predicted by Auzel in the framework of dipole-dipole interaction processes [18]. We compared our results with values obtained from Eq.3 [7] and up to 4 mol\% sample the agreement was within $10 \%$. We believe that the data here reported will be useful to model and design laser in TZN glasses. This measurement method can be easily extended to other type of glasses.

\section{CONCLUSIONS}

A new and simple approach based on the emission spectra measurements was developed to investigate cross-relaxation parameter in Tm-doped samples with very high doping levels. Thanks to this new method we were able to experimentally calculate the cross relaxation parameter of Tm3+ ions in tellurite glasses over a wide range of concentrations. This approach demonstrated to be very sensitive and can be used to investigate other kind of Tm-doped glasses. The obtained values of cross-relaxation show a linear dependence with dopant concentration and value of the linear fit slope was $1.81 \times 10^{-17} \mathrm{~cm}^{-3} \mathrm{~s}^{-1} \% \mathrm{~mol}^{-1}$. The knowledge of this value allows a proper modeling of Tm-doped tellurite glass laser by properly comparing the impact of different doping level.

\section{ACKNOWLEDGEMENTS}

We acknowledge the support by FP7 LIFT project (Leadership in Fiber Technology, Grant \#228587), and the collaboration within COST Action MP0702. R. Balda acknowledges financial support from the Spanish Government under project MAT2009-14282-C02-02.

\section{REFERENCES}

[1] D. C. Hanna, I. M. Jauncey, R. M. Percival, I. R. Perry, R. G. Smart, P. J. Suni, J. E. Townsend, and A. C. Tropper, "Continuous-wave oscillation of a monomode thulium-doped fibre laser," Electron. Lett. 24, 1222-1223 (1988).

[2] S. D. Jackson and A. Lauto, "Diode-pumped fiber lasers: A new clinical tool," Lasers in Surgery and Medicine 30, 184-190 (2002).

[3] J. Y. Allain, M. Monerie, and H. Poignant, "Tunable CW lasing around 0.82, 1.48, 1.88 and $2.35 \mu \mathrm{m}$ in thuliumdoped fluorozirconate fibre," Electron. Lett. 25, 1660-1662 (1989).

[4] E. R. M. Taylor, L. N. Ng, J. Nilsson, R. Caponi, A. Pagano, M. Potenza, and B. Sordo, "Thulium-doped tellurite fiber amplifier," IEEE Photon.Technol. Lett. 16, 777-779 (2004).

[5] M. Yamane and Y. Asahara, "Glasses for photonics" (Cambridge: Cambridge University Press, 2004).

[6] W. Jianfeng, J. Shibin, L. Tao, G. Jihong, N. Peyghambarian, and N. P. Barnes, "Efficient thulium-doped $2 \mu \mathrm{m}$ germanate fiber laser," IEEE Photon.Technol. Lett. 18, 334-336 ( 2006).

[7] A. S. S. de Camargo, S. L. de Oliveira, D. F. de Sousa, L. A. O. Nunes, and D. W. Hewak, "Spectroscopic properties and energy transfer parameters of $\mathrm{Tm}^{3+}$ ions in gallium lanthanum sulfide glass," J. Phys.: Condens. Matter 14, 9495-9505 (2002).

[8] R. R. Petrin, M. G. Jani, R. C. Powell, and M. Kokta, "Spectral dynamics of laser pumped $\mathrm{Y}_{3} \mathrm{Al}_{5} \mathrm{O}_{12}$ : Tm: Ho lasers," Opt. Mater. 1, 111-124 (1992).

[9] C. A. Evans, Z. Ikonic', B. Richards, P. Harrison and A. Jha, "Theoretical Modeling of a $2 \mu \mathrm{m} \mathrm{Tm}^{3+}$ Doped Tellurite Fiber Laser: The Influence of Cross Relaxation,” J. Lightwave Technol. 27, 4026-4032 (2009).

[10] J. Wu, S. Jiang, T. Luo, J. Geng, N. Peyghambarian, and N. P. Barnes, "Efficient thulium-doped 2- $\mu$ m germanate fiber laser," IEEE Photon.Technol. Lett. 18, 334-336 (2006).

[11] Q. Huang, Q. Wang, J. Chang, X. Zhang, Z. Liu, and G. Yu, "Optical parameters and upconversion fluorescence in $\mathrm{Tm}^{3+} / \mathrm{Yb}^{3+}$ co-doped tellurite glass," Laser Physics 20, 865-870 (2010). 
[12] B. Richards, Y. Tsang, D. Binks, J. Lousteau, and A. Jha, "Efficient $2 \mu \mathrm{m}$ doped tellurite fiber laser," Opt. Lett. 33, 402-404 (2008).

[13] C. R. Giles, C. A. Burrus, D. DiGiovanni, N. K. Dutta, and G. Raybon, "Characterization of erbium-doped fibers and application to modeling 980-nm and 1480-nm pumped amplifiers," IEEE Photon. Technol. Lett. 3, 363 - 365 (1991).

[14] C. R. Giles and E. Desurvire, "Propagation of signal and noise in concatenated erbium-doped fiber optical amplifiers," J. Lightwave Technol. 9, 147 - 154 (1991).

[15] H. Gebavi, D Milanese, R Balda, S Chaussedent, M Ferrari, J Fernandez, and M Ferraris, "Spectroscopy and optical characterization of thulium doped TZN glasses," J. of Appl. Phys. 43, 135104-135111 (2010).

[16] H. Gebavi, D. Milanese, G. Liao, Q. Chen, M. Ferraris, M. Ivand, O. Gamulin, and S. Taccheo, "Spectroscopic investigation and optical characterization of novel highly thulium doped tellurite glasses," J. of Non Cryst. Solids $355,548-555(2009)$

[17] S. D. Jackson and T. A. King, "Theoretical Modeling of Tm-Doped Silica Fiber Lasers," J. Lightwave Technol. 17, 948-954 (1999).

[18] F. Auzel, G. Baldacchini, L. Laversenne, and G. Boulon, "Radiation trapping and self-quenching analysis in Yb3+, Er3+, and Ho3+ doped Y2O3," Opt. Mater. 24, 103-109 (2003). 